\title{
MESA: A new configuration for measuring electromagnetic field fluctuations
}

\author{
TIMOTHY M. HARTE \\ ResCare, Inc., Evansville, Indiana \\ DAVID L. BLACK \\ JAM Software, Inc., Ames, Iowa \\ and \\ MICHAEL T. HOLLINSHEAD \\ Sangamon County Data Processing Department, Springfield, Illinois
}

\begin{abstract}
This paper describes how the multi-energy sensor array has been refitted to meet the needs of measuring geomagnetic and other types of electromagnetic phenomena in an environment. This portable laptop computer system was designed to measure the interaction of multiple frequencies with the psychological and physiological processes that underlie human exposure to electromagnetic fields across the spectra. New sensors and analytical software have been implemented in the new configuration.
\end{abstract}

Electromagnetic fields and other energy across the electromagnetic spectra need to be measured not only for their environmental levels and interactions among the different spectra (Savitz, 1993), but also to study the psychological and physiological effects on humans (see, e.g., Gunby, 1995). Electromagnetic fields are being studied for their effects on humans in diseases such as neuroblastoma (e.g., Jones, Shih, Thurston, Ware, \& Cole, 1993), breast cancer (e.g., Gammon et al., 1998), prostate cancer (e.g., Goldberg, 1998), and leukemia (e.g., Goldberg, 1998). They are also being studied as possible beneficial resources for human health issues (Browner, Jupiter, Levine, \& Trafton, 1998), such as facial nerve regeneration (Byers, Clark, \& Thompson, 1998), arthritis (www.ama-assn.org/med$\mathrm{sci} / \mathrm{csa}$ ), multiple sclerosis (www.intergate.bc.ca/enermed), and migraine headaches (Lappin, 1998).

Geomagnetic activity has also been blamed for hallucinatory episodes, especially during REM sleep (see, e.g., Randall \& Randall, 1991). Other researchers think that the sleep states themselves may be the cause of the hallucinatory behavior (e.g., Blackmore, 1998; Nickell, 1997; Persinger, 1993). If the effects of electromagnetic fields on humans are this powerful, the reports of the phenomena should be prevalent within the population. Housing, offices, and human activity near large transformer stations, power lines, and fault lines may play a key role in studies of hallucinatory episodes (Sisir \& Millis, 1986).

Computers are being used to investigate electromagnetic phenomena in the field. The multi-energy sensor

Correspondence concerning this article should be addressed to T. Harte, 5317 Warren Drive, Evansville, IN 47710 (e-mail: tmharte@) juno.com). array (MESA; Houran, Lange, \& Black, 1998) is a portable laptop computer system designed to measure lowfrequency electromagnetic fluctuations in an environment. A previous version of MESA utilized infrared (IR) and visible (Vis) light sensors, $60-\mathrm{Hz}$ magnetometers, and a seismic vibration sensor. This previous system did not measure geomagnetic activity, perhaps the most important of all the frequencies that should be studied in the electromagnetic spectrum.

This paper will describe new sensors that were added to MESA and will discuss software additions, improvements, and some issues about methodology and reporting of the data in scientific journals. The sensors for the new version of MESA are sensitive to IR, ultraviolet (UV), and Vis light, $60-\mathrm{Hz}$ electromagnetic fields, radio/microwave activity, seismic activity, and static $(0-0.5 \mathrm{~Hz})$ electromagnetic fields, where primarily geomagnetic activity may occur. MESA was also fitted for other sensors to measure other frequencies, such as gamma rays, $x$-rays, galvanic skin responses, and temperature readings in an environment.

\section{MESA SYSTEM AND SUPPORT SPECIFICATIONS}

The new configuration of MESA still utilizes IR and Vis light sensors, $60-\mathrm{Hz}$ Tri-Field magnetometers, and a seismic activity device. It adds a UV light sensor, three static electromagnetic field sensors $(0-0.5 \mathrm{~Hz})$, and a temperature sensor. The sensors are still connected to a data acquisition board that has eight channels, which is connected to a portable laptop computer with a larger hard disk drive. The data can now be analyzed directly on the laptop computer, whereas before, a phone link was utilized to upload the data to another computer for analy- 
sis. The data are then displayed as graphs and overlaid on top of one another so one can look for interactions between electromagnetic spectra. Readers are referred to the previous article (Houran et al., 1998) for technical specifications not mentioned here.

\section{New Configuration}

Channel 1. The IR light photoresistor (Radio Shack, No. 276-1657) consists of a single transistor whose base region is sensitive to IR wavelengths. Connections to the base and the emitter terminals of the transistor make it possible to use it as a variable resistance device. The IR transistor's characteristics with regard to light levels are (1) the sensor is most sensitive to IR light (long-wavelength light) regions and (2) the response of the IR sensor is fast, in the nanosecond range. Calibration tests revealed that a $60-\mathrm{Hz}$ flicker source is very easily seen on the IR sensor's output and that the response to light changes is virtually instantaneous.

Channel 2. The UV sensor (Solartech, Inc.; Harrison Township, MI) is both a UVA and a UVB digital sensor that is sensitive to UV light wavelengths. It, too, has an instantaneous time output and operates in the variable resistance mode.

Channel 3. The Vis CdS light photoresistor (Radio Shack, No. 276-1657) operates in the variable resistance mode and is sensitive to Vis light wavelengths. The frequency/sensitivity curve for CdS is very close to that of the human eye. Its time domain response is variable; that is, its output depends on how quickly the intensity of a light source is changing. Minor variations seem to be tracked precisely. For instance, the flicker of a $60-\mathrm{Hz}$ fluorescent light is easily seen on the output of this detector. However, large changes can require up to $2 \mathrm{sec}$ to be accurately reflected in this sensor. The sensor is such that a high voltage results from low ambient light conditions and a low voltage results from high ambient light conditions. A pullup resistor is selected that nets the desired sensitivity.

Channel 4. The vibration/relative seismic activity sensor (Radio Shack, No. 273-091) is a large piezo-electric transducer, which is sold as a speaker element. We have mechanically coupled the device to a 10 -pound weight that functions as a suitable structure that stands by itself. Voltages as high as $+11 \mathrm{~V}$ were measured from the device with moderate levels of compression. High levels of compression presented a problem for the A/D equipment, because overloading of $\mathrm{a}+5-\mathrm{V}$ A/D input resulted in splatter across adjacent channels. However, this problem was solved by placing a $5-\mathrm{V}$ Zener diode across the piezo's terminals so that any output voltages higher than the Zener would shunt to ground.

Channel 5. The Tri-Field meter (AlphaLab, Inc., Salt Lake City) can detect three fields - magnetic, electric, and radio frequencies. The three fields could be sampled simultaneously by fitting more than one meter to the $\mathrm{A} / \mathrm{D}$ box. The meters have two ranges for magnetic fields, $0-3 \mathrm{mG}$ and $0-100 \mathrm{mG}$. These meters measure the RMS value of sinusoidal magnetic fields. This is a limitation of the devices that is designed to measure $\mathrm{AC}$ magnetic fields from 0.2 to $100 \mathrm{mG}$ at $60-\mathrm{Hz}$ frequency. The output voltage for the meter ranges from $0.89 \mathrm{~V}$ for a "zero" indication, to approximately $2.55 \mathrm{~V}$ for a fullscale indication. Calibration tests have shown that this output voltage remains proportional to the external field strength even past the meter's ability to visually indicate the field strength (i.e., when the meter is pegged).

Channel 6. The static sensor pointed east (Speake \& Company Ltd; Murietta, CA) consists of very high sensitivity static magnetic field sensors operating in the \pm 50 microtesla range ( \pm 0.5 oersted). They are simple, essentially three terminal devices, operating from a single $+5-\mathrm{V}$ supply. The output is a robust $5-\mathrm{V}$ rectangular pulse whose period is directly proportional to the field strength (giving it a frequency that varies inversely with the field), making it very easy to interface with a computer or micro controller. Since the range covers the earth's field magnitude, multiple sensors can easily be arranged to provide compass orientation or full three-dimensional orientation, using the local earth's field as a reference guide. These sensors are utilized for gimbaled compasses and virtual reality helmets and to predict earthquake activity. Other field uses might include conventional magnetometry, earth field magnetometry, ferrous metal detectors, internal vehicle reorientation alarm systems, external vehicle or ship passage sensors, wreck finders, noncontact current sensing measurement, conveyor belt sensors or counters in conjunction with small permanent magnets, movement and proximity sensors, and ferrous impurity detectors for nonmagnetic alloys. These sensors could also be used for archaeological artifact assessment and magnetic material measurement.

Channel 7. The static sensor pointed up (Speake \& Company) is utilized for the second-dimensional results of locating and measuring the earth's field in a threedimensional graph of an environment.

Channel 8. The static sensor pointed north (Speake \& Company) is utilized for the third-dimensional results of locating and measuring the earth's field in a three-dimensional graph of an environment.

Placement of the sensors. In the previous version of MESA, the sensors were placed onto a microphone stand, affixed with Velcro tape, with the light sensors on the base and the TriField meters about 5 feet in the air. This has been changed recently by placing the sensors in a wooden box, approximately $2 \times 2 \mathrm{ft}$, with a light source also affixed in the box (see Figure 1). This light source will act as a standard or comparison, to see whether electromagnetic phenomena will interact, or somehow affect, the control light source. Another sampling run will not involve the control light source, as a baseline for the experimental site. The static sensors require a separate amplifier and are placed away from the 


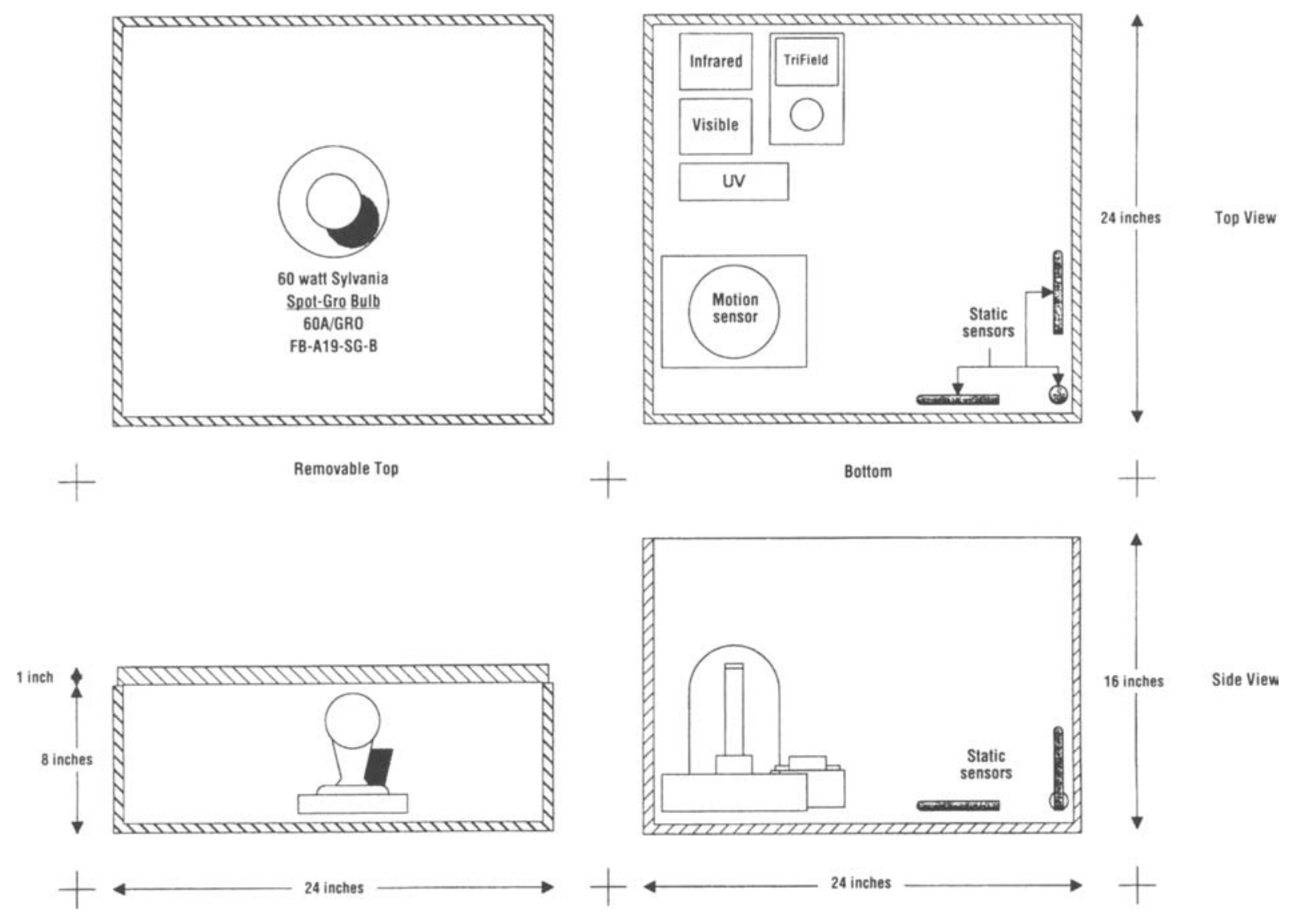

Figure 1. The multi-energy sensor array (MESA).

battery power source, to prevent false readings. Once again, the sensors are easily moved from one location to another by disconnecting the cables and moving the box to another location within a site, if necessary.

\section{Reporting the Data}

Following the collection of the data, a fast Fourier transformations analysis can be implemented and transferred to graphs for further analysis. This analysis program has been installed directly onto the hard drive of the laptop computer. Data files can be analyzed and graphs printed, to examine the measurements of the electromagnetic spectra. Following the collection of the data, several strategies may be employed to improve the stationary qualities of the time-series data.

First, epochs of data that are adequately stationary can be selected from the time-series for spectral analysis and then, subsequently, combined, provided the segments are taken from sites under the same conditions. A test for nonstationarity of time-series data has been developed and tested by Weber, Molenaar, and van der Molen (1992). This method essentially "searches" the time-series information for the longest stationary segments, extracting only those segments, prior to submission for power spectral analysis. Second, the time series data can be mathematically modeled into components, separating nonstationary components, such as aperiodic linear trends, from more stationary ones (Gottman, 1990). An example of this strategy is found in the method of Porges (Porges \& Bohrer, 1990), which essentially generates a new, filtered time-series, presumed to be devoid of undesired elements contributing to the data variability.

Another idea is to analyze the data to see if there is a footprint. Correlation of multiple channels across energy/spectra may show how the various frequencies interact with the human brain or physiological functioning. If there are problematic environments that are alike, what are the psychological, physiological, and physical complaints reported by people in that environment? If environments are different, what type of psychological and physiological symptoms occur in that "different" environment? Environments without these psychological and physical complaints are being measured as well.

Other configurations of MESA may include the introduction of a human subject into an environment. The galvanic skin response of a person could be easily measured, as well as heart rate, respiration, and electric activity of the brain. These sensors could easily be fitted to the present system. More detailed information about MESA can be obtained by contacting the authors. 


\section{CONCLUSION}

MESA has been fitted with more advanced sensors and is quite reliable and precise for the requirements of our research, which include measuring natural places where people have experienced visual, auditory, and olfactory hallucinations, sensed presence and vestibular alterations, had sudden acute emotional responses, and have experienced other anomalous phenomena. MESA can be used to study the psychological and physiological effects in humans caused by electromagnetic energy across the spectrum.

\section{REFERENCES}

BlaCKMORE, S. (1998, May/June). Abduction by aliens or sleep paralysis. Skeptical Inquirer, 22 (3), 23-28.

Browner, B. D., Jupiter, J. B., Levine, A. M., \& Trafton, P. G. (1998). Skeletal trauma: Fractures, dislocations, and ligamentous injuries (Vols. 1 and 2). Available: www.ama-assn.org

Byers, J. M., Clark, K. F., \& Thompson, G. C. (1998). Effect of pulsed electromagnetic stimulation on facial nerve regeneration. Archives of Otolaryngology: Head \& Neck Surgery, 124, 383-389.

Gammon, M. D., Schonberg, J. B., Britton, J. A., Kelsey, J. L. Stanford, J. L., Malone, K. E., Coates, R. J., Brogan, D. J., Totischman, N., Swanson, C. A., \& Brinton, L. A. (1998). Electric blanket use and breast cancer among younger women. American Journal of Epidemiology, 148, 556-563.

GoldberG, R. J. (Ed.) (1998). Cancer Web. Available: http://infoventures.com/cancer

GotTman, J. (1990). Time-series analysis applied to physiological data In J. T. Cacioppo \& L. G. Tassinary (Eds.), Principles of psychophysiology: Physical, social, and inferential elements (pp. 754-774). New York: Cambridge University Press.

GunBY, P. (1995). Unique medical problems of military become part of intensified research into women's health. Journal of the American Medical Association: Medical News \& Perspectives, 12, 932-933.

Houran, J., Lange, R., \& Black, D. L. (1998). MESA: A portable multi-energy sensor array for low-frequency electromagnetic field fluctuations. Behavior Research Methods, Instruments, \& Computers, 30, 411-415.

Jones, T. L., Shih, C. H., Thurston, D. H., Ware, B. J., \& Cole, P. (1993). Selection bias from differential residential mobility as an explanation for association of wire codes with childhood cancer. Journal of Clinical Epidemiology, 46, 545-548.

LAPPIN, M. S. (1998) Analysis of 262 migraine patients. Available: http://www.intergate.bc.ca/business/enermed

NiCKELL, J. (1997, May/June). Alien abductions as sleep related phenomenon. Skeptical Inquirer, 22 (3), 16-18.

Persinger, M. A. (1993). Average diurnal changes in melatonin levels are associated with hourly incidence of bereavement apparitions: Support for the hypothesis of temporal (limbic) lobe microseizuring. Perceptual \& Motor Skills, 76, 444-446.

Porges, S. W., \& Bohrer, R. E. (1990). The analysis of periodic processes in psychophysiological research. In J. T. Cacioppo \& L. G. Tassinary (Eds.), Principles of psychophysiology: Physical, social, and inferential elements (pp. 708-753). New York: Cambridge University Press.

Randall, W., \& Randall, S. (1991). The solar wind and hallucinations: A possible relation to magnetic disturbances. Bioelectromagnetics, 12, 67-70.

SAvitz, D. A. (1993). Overview of epidemiologic research on electric and magnetic fields and cancer. American Industrial Hygiene Association Journal, 54, 197-204.

SisiR, K., \& MiluIs, R. M. (Eds.) (1986). Biological effects of electropollution. Philadelphia: Information Ventures.

Weber, E. J. M., Molenaar, P. C. M., \& van der Molen, M. W. (1992). A non-stationarity test for the spectral analysis of physiologic time series with an application to respiratory sinus arrythmia. Psychophysiology, 29, 55-65.

(Manuscript received April 2, 1999; revision accepted for publication May 17, 1999.) 